\title{
Maximising the value of missing data
}

Received (in revised form): 15th October, 2004

\begin{abstract}
Atai Winkler
studied chemical engineering at Imperial College of Science and Technology, and obtained a first class honours degree. He then stayed at Imperial College to study for his $\mathrm{PhD}$ and it was during this period that he became interested in statistics. His skills and experience are in demand forecasting and the statistical analysis and modelling of databases. In 2002 he set up Atlantec Software with a colleague to develop advanced business-oriented statistical software in the areas of database analysis and retail demand forecasting and analysis.
\end{abstract}

\section{Paul McCarthy}

is Managing Director of McCarthy O'Connor, below-the-line media specialists, providing data research, planning, data/list provision and data management. He is also Managing Director of MOC Evolution, a customer insight and data planning consultancy, providing customer relationship management (CRM) analytics and data-driven return on investment (ROI) planning.

\begin{abstract}
The subject of missing values in databases and how to handle them has received very little attention in the statistics and data mining literature ${ }^{1-3}$ and even less, if any at all, in the marketing literature. The usual attitude of practitioners is 'we'll just have to ignore records with missing values'. On the other hand, a few very advanced theoretical solutions have been developed, some of which have been applied, particularly to clinical trials data. These solutions can only be applied to small databases, not to the very large databases held by many companies on their customers. This paper describes a new method for imputing missing values in such very large databases. Two particular features of the method are that it can handle all combinations of variable type (continuous, ordinal and categorical) and that all the missing values in the database are imputed in one run of the software. It is based on the k-nearest neighbours method, a well-known method in data mining. The paper concludes by presenting the results of a study of this method when used to impute the missing values in a real set of data.

This paper is only concerned with 'missing' data, ie data that are not known but which have real values. It does not address the problem of 'empty' data, ie data that are not known but which cannot have real values.
\end{abstract}

Atai Winkler Wintech Associates, 37 The Crosspath, Radlett, Herts, WD7 8HR, UK.

Tel: $+44(0) 1923852701$; Fax: $+44(0) 1923852701$; e-mail:

Atai.winkler@win-tech.co.uk

\section{INTRODUCTION}

Marketers are always striving to develop effective marketing campaigns by maximising the benefits they can achieve from the data they hold in their databases. The results of different campaigns can then be assessed by comparing the return on investment of the campaigns. Whatever the nature and aims of the campaigns, they always start with some form of analysis to gain customer insight, and the results of this analysis lead to segmentation and targeting of potential customers.

As companies move through the cycle of customer acquisition to customer retention, the ability to analyse the data they hold on their customers becomes 
increasingly important. This task, however, is often hampered by the fact that the vast majority of databases have missing information, sometimes called gaps or holes. This may be for historical reasons where the emphasis was on customer acquisition or it may be due to changes in the needs of the organisation over time. Whatever the cause, missing data are a very common and serious problem - it is not uncommon for collected lifestyle data to have 30-40 per cent of their values missing. This is a real problem when the data are to be analysed - if the data are not there, they cannot be analysed either at record level or for the database overall. These missing values mean that the database is not as large or as rich in information as may be assumed from its overall size (number of fields and number of records). Thus, the effect of the missing data is to limit the amount and quality of new information that can be learnt from the database.

With respect to customer retention programmes and CRM, missing customer data will have a serious adverse impact on the outcome of the campaigns because they will be based on incomplete data and therefore weak analysis. The consequence of this is that the segmentation and targeting will be less accurate than they would be if the database were fully populated. This problem of missing data has also heavily affected the lifestyle data sellers who are trying to present a more complete picture of the UK adult population. Since fewer data are now acquired directly from individuals about their demographics as consumers, there is a greater reliance on modelled data. Unfortunately, these modelled data are often obtained from incomplete data. This just compounds the problem of missing data because any bias in the available data manifests itself, and is probably increased, in the modelled data. Thus, a vicious circle of partial data being used to obtain more partial data is started, and after this process has been repeated many times it is likely that the final data bear little resemblance to the true UK population.

Since the problem of missing values in both customer and lifestyle databases is widespread and getting worse, methods that give more accurate estimates of the missing values compared to those obtained using currently available methods have an important and significant contribution to play in improving marketing effectiveness. The desired result of all these methods is a fully populated database with all the missing values replaced by estimates of their true values - a process known as (missing value) imputation. The imputed values should be 'good' and plausible estimates of the true values so that the statistical properties of the fully and partially populated databases are as similar as possible.

\section{MISSING DATA AND EMPTY DATA}

It may be thought that all missing data can and should be imputed. This is not always the case because some data may be missing because they cannot have real values — such values must remain missing. Thus, it is important to understand the difference between 'missing' data and 'empty' data. A value that is not known but which has a real value is a 'missing' value. A value that is not known but which cannot have a real value is an 'empty' value. Therefore, missing data can be imputed but empty data cannot be imputed.

\section{IMPUTATION METHODS}

A number of imputation methods are available, and some of the factors that 
help determine a suitable method are:

— the method's suitability for small databases and large databases;

- whether the method is 'local' or 'global'. In a local method the missing values in each record are imputed at record level but in a global approach the missing values in each record are imputed from the summary statistics of a group of records. This is analogous to the difference between a micro and macro approach;

- whether the method is simple or complicated;

- whether the method is very sensitive to assumptions about the data or whether any assumptions can be relaxed a little;

- whether the method is very demanding in terms of time and cost or whether it requires few resources.

\section{Desirable properties of imputation methods}

Some desirable properties of a 'good' and practical imputation method are:

— its ease of use and minimum user-intervention;

- it should only use the information in the database (no external data are required);

- it is very amenable for use on very large databases;

- all the missing values in all the fields are imputed in one run;

- the order of the records does not affect the imputed values;

- it uses all the known information in each record to impute the missing values in that record;

- the missing values in correlated variables can be imputed;

- the heterogeneity of the database is maintained;

— all the imputed values are plausible;
- variables in different units are allowed;

- it allows all combinations of continuous, ordinal and text variables;

- the size of the database does not affect the search and imputation methods.

\section{Possible methods}

Possible imputation methods include:

- case deletion;

- mean or mode substitution;

- cold deck substitution;

- hot deck substitution;

- regression;

- EM algorithm;

- structural models;

- $k$-nearest neighbours.

\section{Case deletion}

Case deletion avoids rather than solves the problem of missing values because it ignores all the incomplete records. Very often it is the default method of handling missing data. Although very easy to implement, two immediate and severe disadvantages of the method are first that a very large proportion of the records may be ignored and secondly that the remaining records may not be representative of the population. The commercial and financial implications of this bias in the data that are actually analysed are easy to imagine.

Mean substitution and cold deck imputation

Mean substitution and cold deck imputation are two frequently used methods. Mean substitution involves replacing all the missing values in each field by the field's mean or mode as appropriate, and in cold deck imputation the missing values are replaced by external constants, one for each field. These methods are easy and quick to implement but being global methods 
they are very unlikely to maintain the statistical properties of the database. In the case of mean substitution, the mean (mode) values of the fields in the partially and fully populated databases are, by definition, the same, but the variation of each field in the fully populated database is much smaller than the corresponding variation in the partially populated database. The result is that the records are not as clearly differentiated as they should be and so it is harder to understand how people's individual characteristics determine their actions and behaviour from a database which has been fully populated in either of these ways.

Another major problem with mean substitution and cold deck imputation is that unrealistic or even impossible values can be easily imputed. This is because the value imputed in any one field is the mean of the known values in that field. Therefore, if a database contains people across a wide range of age, income and lifestyle attributes and the data can be segmented into a finite number of homogeneous clusters with high intercluster heterogeneity, the mean value of any field across all clusters does not have meaning or significance for any single cluster or all the clusters.

Therefore, using values imputed in this way as the basis for marketing campaigns and other commercial activities may not yield the desired outcomes because the targeting and segmentation are based on poor quality data.

\section{Hot deck imputation}

A slightly more advanced method of imputation is hot deck imputation. This is similar to cold deck imputation except that the missing values are replaced by values from 'similar' records in the database. These similar records are obtained by clustering the complete records and then assigning a cluster to each incomplete record. The missing values in each incomplete record are replaced by values calculated from its associated cluster. Like mean substitution and cold deck imputation, hot deck imputation is a global method.

\section{Regression}

In regression imputation the missing values are replaced by values calculated from a regression equation, for example

$$
y=a+b x_{1}+c x_{2}
$$

$y$ is the variable to be imputed, and $x_{1}$ and $x_{2}$ are other variables $(a, b$ and $c$ are known constants).

Implicit in using (1) is that the values of the variables on the right hand side of it $\left(x_{1}\right.$ and $\left.x_{2}\right)$ in records whose values of $y$ are to be imputed are known. This problem can be overcome by developing the models only from complete records - but this raises a fundamental problem, namely what happens if the complete records are either a small proportion of the database or they are a distinct group in the database rather than being a fair reflection of the database as a whole? On the other hand, regression imputation is a local method because the missing values in each record are calculated from the data in that record - a significant advantage. Notwithstanding this advantage, regression imputation has a number of practical and theoretical problems, including the following:

- since a regression equation must be developed for each variable with missing values, regression imputation is very time consuming, especially in large databases and in databases many of whose fields have missing values;

- working out the equations may be difficult, not least because the correlations between the variables may be weak; 
— different relationships may exist for different homogeneous groups in the database so trying to find one relationship across all groups will yield an unsatisfactory compromise that is not an accurate portrayal of the relationship in any one group. The single equation will predict values that do not reflect any individual's unique characteristics, and so the same problems as those associated with mean substitution, namely reduction in the heterogeneity of the database, may arise;

- the relationships between the variables are artificially and falsely inflated because the missing values are estimated by substituting into the regression equations.

\section{EM algorithm and structural models}

These methods are very advanced and demanding in terms of the time and expertise required. They are not amenable for use on large databases.

\section{$k$-nearest neighbours and imputation}

$k$-nearest neighbours is a data mining method used in estimation and classification problems. Unlike many other methods used in statistical data analysis and modelling, it does not require a model to be developed for each field. Rather, it is based on the simple concept that the (statistical) similarity between two records is calculated from the multivariate distance between them. If two records are similar, ie their corresponding fields have similar values, they will be close to one another and so the distance between them will be 'small' when their common known data are plotted. These records are more similar to one another than are other records with larger distances between them. This geometric way in which the most similar records are found explains why the method is called $k$-nearest neighbours. Thus, the method involves mapping all the data into multidimensional space and then calculating the distances between all pairs of records (each dimension is a variable).

The method works by first finding a pool of donors, ie complete records, for each recipient, ie incomplete record. It then uses the donors' values for each field that is missing in the recipient to impute the missing data in the recipient. There are three stages to the method.

For each incomplete record:

1 search the entire database for similar complete records using the values in the selected fields in the incomplete record;

2 rank the complete records by distance to the incomplete record;

3 use the specified number of complete records in the ranked set to impute the missing values in the incomplete record.

By using all the known data in each recipient to search for its most similar donors and then using these donors to impute the recipient's missing values, the statistical properties of the partially populated database are maintained. This process of searching for similar donors and then using them to impute the missing values is repeated for each recipient. This recipient-by-recipient approach means that each recipient has its own donors, ie nearest neighbours (NNs), from which its missing values are imputed. It is this feature of $k$-nearest neighbours that helps maintain the heterogeneity of the database.

This very localised approach to imputation is in marked contrast to global methods where the imputation is based on groups of recipients and each group has the same donors and therefore the same imputed values. Thus, the variation of the variables in 
Table 1: An example of a partially populated file

\begin{tabular}{rllllllll}
\hline $\begin{array}{l}\text { Record } \\
\text { number }\end{array}$ & $\begin{array}{l}\text { Mar } \\
\text { stat }\end{array}$ & $\begin{array}{l}\text { Res } \\
\text { stat }\end{array}$ & $\begin{array}{l}\text { Age } \\
\text { (mths) }\end{array}$ & $\begin{array}{l}\text { Bank } \\
\text { (mths) }\end{array}$ & $\begin{array}{l}\text { Cheq } \\
\text { card }\end{array}$ & $\begin{array}{l}\text { Add } \\
\text { (mths) }\end{array}$ & $\begin{array}{l}\text { Emp } \\
\text { (mths) }\end{array}$ & Occup \\
\hline 1 & M & T & 334 & 18 & Y & 20 & 12 & ES \\
2 & M & T & 308 & 24 & Y & 24 & 66 & ES \\
3 & $\mathrm{D}$ & & 317 & & N & 36 & & EO \\
4 & M & T & 271 & 60 & N & 36 & 60 & EM \\
5 & M & & & 132 & Y & & 0 & S \\
6 & $\mathrm{D}$ & $\mathrm{T}$ & 516 & 72 & $\mathrm{~N}$ & 6 & 11 & EB \\
7 & $\mathrm{~S}$ & $\mathrm{P}$ & 314 & 14 & $\mathrm{~N}$ & 54 & 42 & SB \\
8 & & & 338 & 12 & & & 66 & EP \\
9 & $\mathrm{M}$ & $\mathrm{Z}$ & 448 & 126 & $\mathrm{Y}$ & 82 & 120 & \\
\hline
\end{tabular}

a database which has been fully populated using a global method is lower than it is in a database which has been fully populated using $k$-nearest neighbours. Furthermore, since each recipient record is treated individually, the method obtains the most accurate imputed values for each recipient record rather than attempting to obtain the most accurate average imputed values across a group of records.

The main reason for the limited use up to now of the $k$-nearest neighbours method with very large databases is that the number of distances that have to be stored and then ranked made it impractical to use on such databases. This problem has now been overcome so that it does not store the distance from the incomplete record being processed to each complete record, rank all the distances and then select the specified number of NNs. This means that only a fraction of the number of complete records in the database are stored at any one time.

A good example of the type of data that can be imputed using $k$-nearest neighbours is lifestyle data. However, variables such as ownership of pets, type of credit card owned and participation in hobbies, for example stamp collecting, should not be imputed because they are generally independent of other variables and do not define people.
To show how $k$-nearest neighbours works, consider the data in Table 1 . The data come from a survey and the fields are:

$$
\begin{array}{ll}
\text { mar_stat: } & \begin{array}{l}
\text { marital status: D } \\
\text { (divorced); M (married); S }
\end{array} \\
\text { (single) } & \begin{array}{l}
\text { residential status: P } \\
\text { (owner-occupier); T (rent } \\
\text { alone); Z (multiple rent) }
\end{array} \\
\text { age: } & \begin{array}{l}
\text { age (months) } \\
\text { time with bank (months) }
\end{array} \\
\text { bank: } & \text { own a cheque guarantee } \\
\text { cheq_card: } & \text { own: N (no); Y (yes) } \\
\text { add: } & \begin{array}{l}
\text { time at current address } \\
\text { (months) }
\end{array} \\
\text { emp: } & \begin{array}{l}
\text { time with current } \\
\text { employer (months) }
\end{array} \\
\text { occup: } & \begin{array}{l}
\text { occupation code } \\
\end{array}
\end{array}
$$

The small database in Table 1 has ten records of which four (records 3, 5, 8 and 10) have missing values and the other six are complete. The three NNs for each incomplete record were calculated using $k$-nearest neighbours and are shown in Table 2 .

Table 2 shows that the three NNs for record 3 are records 4,7 and 2, with record 4 being the most similar and record 2 the least similar of the three NNs. Since there are six complete records in the database, there can be up to six NNs. 
Table 2: The three nearest neighbours for Table 1

\begin{tabular}{clll}
\hline Recipient record & Nearest neighbour & $\begin{array}{l}\text { Next nearest } \\
\text { neighbour }\end{array}$ & $\begin{array}{l}\text { Next nearest } \\
\text { neighbour }\end{array}$ \\
\hline 3 & 4 & 7 & 2 \\
5 & 1 & 2 & 4 \\
8 & 2 & 7 & 4 \\
10 & 9 & 1 & 2 \\
\hline
\end{tabular}

Table 3: The fully populated form of Table 1

\begin{tabular}{rllllllll}
\hline $\begin{array}{l}\text { Record } \\
\text { number }\end{array}$ & $\begin{array}{l}\text { Mar } \\
\text { stat }\end{array}$ & $\begin{array}{l}\text { Res } \\
\text { stat }\end{array}$ & $\begin{array}{l}\text { Age } \\
\text { (mths) }\end{array}$ & $\begin{array}{l}\text { Bank } \\
\text { (mths) }\end{array}$ & $\begin{array}{l}\text { Cheq } \\
\text { card }\end{array}$ & $\begin{array}{l}\text { Add } \\
\text { (mths) }\end{array}$ & $\begin{array}{l}\text { Emp } \\
\text { (mths) }\end{array}$ & Occup \\
\hline 1 & M & T & 334 & 18 & Y & 20 & 12 & ES \\
2 & M & T & 308 & 24 & Y & 24 & 66 & ES \\
3 & $\mathrm{D}$ & $\mathrm{T}$ & 317 & 33 & $\mathrm{~N}$ & 36 & 56 & EO \\
4 & $\mathrm{M}$ & $\mathrm{T}$ & 271 & 60 & $\mathrm{~N}$ & 36 & 60 & EM \\
5 & $\mathrm{M}$ & $\mathrm{T}$ & 304 & 132 & $\mathrm{Y}$ & 27 & 0 & ES \\
6 & $\mathrm{D}$ & $\mathrm{T}$ & 516 & 72 & $\mathrm{~N}$ & 6 & 11 & S \\
7 & $\mathrm{~S}$ & $\mathrm{P}$ & 314 & 14 & $\mathrm{~N}$ & 54 & 42 & EB \\
8 & $\mathrm{M}$ & $\mathrm{T}$ & 338 & 12 & $\mathrm{~N}$ & 38 & 66 & SB \\
9 & $\mathrm{M}$ & $\mathrm{Z}$ & 448 & 126 & $\mathrm{Y}$ & 82 & 120 & EP \\
10 & $\mathrm{M}$ & $\mathrm{T}$ & 749 & 56 & $\mathrm{Y}$ & 12 & 66 & ES \\
\hline
\end{tabular}

If only one donor (the NN) is to be used, the missing values in records 3,5 , 8 and 10 are copied directly from the corresponding fields in records $4,1,2$ and 9 respectively. If two $\mathrm{NNs}$ are to be used, the missing values in records 3,5 , 8 and 10 are calculated from the corresponding fields in records 4 and 7 , 1 and 2, 2 and 7, and 9 and 1 respectively. If three NNs are to be used, the missing values in record 3 are calculated from the corresponding fields in records 4, 7 and 2, record 5 from records 1,2 and 4 , record 8 from records 2, 7 and 4, and record 10 from records 9,1 and 2 .

The fully populated database shown in Table 3 was obtained by using the three NNs shown in Table 2.

As with many methods of data analysis, the trade-off between run-time and accuracy must be considered - a big increase in run-time is not always accompanied by a significant improvement in accuracy. For $k$-nearest neighbours an obvious question is how the number of donors affects the accuracy of the imputed values. It is reasonable to assume that as the number of donors increases, the 'better' will be the imputed values. Increasing the number of donors has, however, two adverse effects: first, as the distance between the recipient and the donors increases, the donors become more dissimilar from the recipient; and secondly, the run-time increases. This and other questions are discussed later in this paper.

\section{EXAMPLE OF USING $\boldsymbol{k}$-NEAREST NEIGHBOURS FOR IMPUTATION}

The use of the $k$-nearest neighbours method for imputation was tested on a database of 20,000 records, of which 13,303 (66.5 per cent) were fully populated and the remaining 6,697 (33.5 per cent) had at least one missing value. The actual values of these missing values were known so that after the imputation the imputed values could be compared 
Table 4: The distribution of missing values

\begin{tabular}{lrl}
\hline $\begin{array}{l}\text { Number } \\
\text { missing }\end{array}$ & $\begin{array}{l}\text { Number } \\
\text { records }\end{array}$ & $\begin{array}{l}\text { Cumulative } \\
\%\end{array}$ \\
\hline 1 & 2,947 & 44.00 \\
2 & 2,442 & 80.47 \\
3 & 1,026 & 95.79 \\
4 & 238 & 99.34 \\
5 & 40 & 99.94 \\
6 & 3 & 99.99 \\
7 & 1 & 100.00 \\
8 & 0 & 100.00 \\
\hline
\end{tabular}

with the actual values. The results of this comparison are presented. The imputation and validation were carried out on a Dell Precision 650 Workstation with 1 Xeon $3.2 \mathrm{GHz}$ processor and $3 \mathrm{~Gb}$ RAM. The distribution of missing values in the 6,697 records is shown in Table 4. The table had eight fields, as described in Table 5. Four runs were carried out. The settings of the runs are shown in Table 6.

\section{IMPUTATION RESULTS}

The results of the imputation for the text variables are shown in Table 7.

Age was the only continuous field and its null count was 4,027. The results of the four runs are shown in Table 8. (ME stands for Mean Error and MAE stands for Mean Absolute Error.) Income was the only ordinal field. It was divided into ten levels, and its null count was 1,255. The results of the four runs are shown in Table 9 as the percentage of entries in cells off the Leading Diagonal (LD) in the cross-classification matrix (this is just

Table 5: The fields

\begin{tabular}{lll}
\hline Field & Description & Type \\
\hline p_hhld & head of household & text \\
h_comp & household composition & text \\
h_shrs & value of shares held & text \\
h_prop & type of property & text \\
h_inc & household income & ordinal \\
h_res & residence type & text \\
_ten & household tenure & text \\
age & age & continuous \\
\hline
\end{tabular}

Table 6: The imputation setting

\begin{tabular}{llll}
\hline Run Number & $\begin{array}{l}\text { Sampling } \\
\text { percentage }\end{array}$ & $\begin{array}{l}\text { Number of complete } \\
\text { records sampled }\end{array}$ & $\begin{array}{l}\text { Number of nearest } \\
\text { neighbours }\end{array}$ \\
\hline 1 & 10 & 1,300 & 10 \\
2 & 10 & 1,300 & 20 \\
3 & 5 & 665 & 10 \\
4 & 5 & 665 & 1 \\
\hline
\end{tabular}

Table 7: The imputation results (text variables)

\begin{tabular}{lccllll}
\hline Field & Null count & $\begin{array}{l}\text { Number of } \\
\text { categories }\end{array}$ & $\begin{array}{l}\text { \% Correctly } \\
\text { imputed } \\
\text { (run 1) }\end{array}$ & $\begin{array}{l}\text { \% Correctly } \\
\text { imputed } \\
\text { (run 2) }\end{array}$ & $\begin{array}{l}\text { \% Correctly } \\
\text { imputed } \\
\text { (run 3) }\end{array}$ & $\begin{array}{l}\text { \% Correctly } \\
\text { imputed } \\
\text { (run 4) }\end{array}$ \\
\hline p_hhld & 991 & 2 & 90.62 & 90.41 & 90.31 & 88.50 \\
h_comp & 1,090 & 12 & 37.43 & 35.23 & 33.94 & 30.83 \\
h_shrs & 1,090 & 3 & 76.97 & 77.34 & 76.79 & 75.32 \\
h_prop & 1,189 & 5 & 83.52 & 83.35 & 82.51 & 79.14 \\
h_res & 1,255 & 5 & 44.94 & 45.10 & 45.82 & 40.24 \\
h_ten & 1,189 & 3 & 68.04 & 66.69 & 68.29 & 60.64 \\
\hline
\end{tabular}


Table 8: The imputation result (continuous variables)

\begin{tabular}{lll}
\hline Run number & ME & MAE \\
\hline 1 & 0.64 & 12.69 \\
2 & 1.15 & 12.82 \\
3 & 1.07 & 12.90 \\
4 & 0.71 & 14.21 \\
\hline
\end{tabular}

a crosstab of actual values against imputed values). Table 10 shows the run-times of the four runs.

\section{DISCUSSION OF RESULTS}

Table 7 shows that the percentage of correctly imputed text variables is not very sensitive to the sampling percentage or to the number of NNs. This is because the records are randomly distributed in the database rather than there being a number of distinct and homogeneous groups in the database each of which is concentrated in adjoining records, and the variation of the fields in the NNs hardly increases as the number of NNs increases.

It is expected that as the number of $\mathrm{NNs}$ increases the variation of the fields in the NNs would also increase because the (complete) records in the search domains are ranked by similarity to the incomplete record. This means that the record most recently added to the search domain is always most similar to the previously added record and least similar to the first record in the search domain. If the variation of the fields in the NNs were much greater, the success of the imputation would be much more sensitive to the number of NNs. In general, the rate at which the variation of the fields in the NNs increases depends on the data and the number of NNs.

Another interesting result in Table 7 is the variation of the percentage of values correctly imputed across the fields; for example in run 1 it is between 37.43 and 90.62. Now, it is reasonable to assume that as the number of categories of a text field increases, the percentage of values correctly imputed would decrease (all other things being equal). There is, however, another more important factor at play, and that is the standard deviation of the relative frequencies of the categories. Table 11 shows this standard deviation, the rank of the standard deviations, the rank of the percentage of values correctly imputed for any of the four runs in Table 7 and the rank of the number of categories for all the text fields ( 1 is the smallest rank and 6 is the largest rank).

There is an almost perfect one to one correspondence between the third and fourth columns in Table 11. The only discrepancy occurs with the ranks of h_shrs and h_prop — their standard deviations are more similar to one another than are other pairs of standard deviations. This suggests that the variation in the relative frequencies of the categories of the fields is a significant factor in determining the percentage of values correctly imputed. The fourth and fifth columns in Table 11 appear, however, to follow a weak inverse

Table 9: The imputation results (ordinal variables)

\begin{tabular}{llllllllll}
\hline $\begin{array}{l}\text { Run } \\
\text { number }\end{array}$ & $\begin{array}{l}\text { Correctly } \\
\text { imputed }\end{array}$ & $\begin{array}{l}\% 1 \\
\text { off LD }\end{array}$ & $\begin{array}{l}\% 2 \\
\text { off LD }\end{array}$ & $\begin{array}{l}\% 3 \\
\text { off LD }\end{array}$ & $\begin{array}{l}\% 4 \\
\text { off LD }\end{array}$ & $\begin{array}{l}\% 5 \\
\text { off LD }\end{array}$ & $\begin{array}{l}\% 6 \\
\text { off LD }\end{array}$ & $\begin{array}{l}\% 7 \\
\text { off LD }\end{array}$ & $\begin{array}{l}\% \text { LD } \\
\text { Loff }\end{array}$ \\
\hline 1 & 17.53 & 32.91 & 25.02 & 14.02 & 6.93 & 2.63 & 0.72 & 0.24 & \\
2 & 17.05 & 31.39 & 24.86 & 16.10 & 7.09 & 2.71 & 0.80 & & \\
3 & 15.62 & 31.08 & 23.59 & 16.02 & 8.84 & 3.82 & 0.80 & 0.23 & 0.32 \\
4 & 17.13 & 29.72 & 20.88 & 15.30 & 9.24 & 4.46 & 2.07 & 0.88 & 0.32 \\
\hline
\end{tabular}


Table 10: The imputation run-times

\begin{tabular}{ll}
\hline Run number & Run-time (minutes) \\
\hline 1 & 47 \\
2 & 48 \\
3 & 24 \\
4 & 24 \\
\hline
\end{tabular}

relationship - the larger the number of categories a text field has, the smaller is its percentage of values correctly imputed likely to be.

The results in Table 8 show that for none of the runs is the absolute value of the mean error equal to the mean absolute error. This is because the values of age in the NNs are not all positively or all negatively biased, ie each set of NNs has values of age both above and below the true values. Once again, this shows that the records are randomly distributed in the database.

The results in Table 9 appear to suggest that the imputed ordinal values are biased because the values in the \% 1 off LD, \% 2 off LD and \% 3 off LD columns are mostly greater than the corresponding values in the \% Correctly imputed column. This impression is incorrect because the entries in each column are obtained by adding a different number of values. Table 12 shows the number of values used to calculate the entries in each position in Table 9. Thus, for run 117.53 was obtained by adding ten numbers, 32.91 was obtained by adding 18 numbers and 25.02 was obtained by adding 16
Table 12: The number of entries used in the cross-classification matrix (ordinal variables)

\begin{tabular}{ll}
\hline Position & Number of values \\
\hline LD & 10 \\
1 off LD & 18 \\
2 off LD & 16 \\
3 off LD & 14 \\
4 off LD & 12 \\
5 off LD & 10 \\
6 off LD & 8 \\
7 off LD & 6 \\
8 off LD & 4 \\
9 off LD & 2 \\
\hline
\end{tabular}

numbers. One approximate way of determining if the imputed values are biased is to normalise each entry in Table 9 by dividing it by the number of values for that position as shown in Table 12 .

Table 9 shows that for all the runs about half the values were imputed correctly or within one level either side of the LD, and that the percentages fall off very quickly as the cells move away from the LD.

Comparing Tables 6 and 10, it is immediately apparent that the run-time is strongly influenced by the number of complete records from which the imputed values are calculated and not affected at all by the number of NNs. This is as expected because the number of distances calculated is given by the product of the number of incomplete records and the number of complete records used. The number of NNs does not determine the run-time because the software used in this study has a very powerful sorting algorithm which sorts

Table 11: The standard deviation of the relative frequencies of the categories (text variables)

\begin{tabular}{lllll}
\hline Field & $\begin{array}{l}\text { Standard } \\
\text { deviation }\end{array}$ & $\begin{array}{l}\text { Rank of standard } \\
\text { deviation }\end{array}$ & $\begin{array}{l}\text { Rank of \% } \\
\text { correctly imputed }\end{array}$ & $\begin{array}{l}\text { Rank of number } \\
\text { of categories }\end{array}$ \\
\hline p_hhld & 57.13 & 6 & 6 & 1 \\
h_comp & 9.46 & 1 & 1 & 6 \\
h_shrs & 39.78 & 5 & 4 & 2.5 \\
h_prop & 34.31 & 4 & 5 & 4.5 \\
h_res & 11.47 & 2 & 2 & 4.5 \\
h_ten & 28.49 & 3 & 3 & 2.5 \\
\hline
\end{tabular}


the distances as they are processed. This means that the number of distances actually stored as each incomplete record is processed is kept to a minimum and is very close to or equal to the number of NNs specified at input. The alternative solution to this sorting problem is to store the distance for each complete record and then when all the complete records have been processed rank all the distances from smallest to largest. This approach has two big computational problems: first, the larger the database the more distances have to be stored; and secondly, the time required to sort these distances is not insignificant, and indeed may be greater than the time required to calculate the distances and impute the missing values.

\section{CONCLUSION}

This paper has presented the results of a study on how a powerful data mining technique, $k$-nearest neighbours, has been enhanced and then applied to the ever-present problem of how to impute missing values in large databases. The enhancements make the method amenable for use on very large commercial databases. The results of a study presented in this paper show that its overall accuracy is very high. The advantage of having more accurate imputed data is that campaigns can be better targeted. In turn, this will generate higher response rates and a greater return on investment.

\section{References}

1 Manly, B. (1994) 'Multivariate statistical methods: A primer', Chapman and Hall, London, UK.

2 Batista, G. E. A. P. A. and Monard, M. C. (2002) 'K-nearest neighbour as imputation method: Experimental results', technical report ICMC-USP (University of Sao Paulo), ISSN-0103-2569.

3 Batista, G. E. A. P. A. and Monard, M. C. (2003) 'An analysis of four missing data treatment methods for supervised learning', Applied Artificial Intelligence, Vol. 17, Nos. 5/6, pp. 519-533. 